\title{
ORBITS OF INTERPLANETARY DUST PARTICLES INSIDE 1 AU AS OBSERVED BY HELIOS
}

\author{
E. Grün, H. Fechtig and J. Kissel \\ Max-Planck-Institut für Kernphysik \\ Postfach 103980 \\ D - 6900 Heidelberg \\ F.R.G.
}

\begin{abstract}
The in situ dust detectors on board the Helios spaceprobes detected impacts of micrometeoroids between $0.3 \mathrm{AU}$ and $1 \mathrm{AU}$ distance from the sun. Among the measured quantities were mass, impact speed and flight direction of the dust particles. Radial variations of the flux, azimuthal distribution and impact speed are discussed. Significantly different results were obtained for the "apex" particles observed by both ecliptic and south sensors and for the "eccentric" particles detected only by the south sensor. The radial spatial density variation of the "eccentric" particles is compatible with that derived for zodiacal light particles. Whereas the spatial density of "apex" particles peaks at 0.5 to $0.6 \mathrm{AU}$. From the measured quantities probability distributions of orbital elements were derived for the observed micrometeoroids. About $60 \%$ of the observed micrometeoroids ("apex" particles) are on low energy orbits ( $a \leqslant 0.6 \mathrm{AU}$ ). About $30 \%$ of the particles ("eccentric" particles) had high energy orbits (a $\gtrsim 0.9 \mathrm{AU}$ ). More than $10 \%$ of the observed particles show high probabilities for travelling on hyperbolic orbits.
\end{abstract}

\section{INTRODUCTION}

The Helios spacecrafts orbit the sun between $0.3 \mathrm{AU}$ and $1 \mathrm{AU}$. We report on data from the micrometeoroid detector taken during the first 10 orbit revolutions of Helios 1. Preliminary data from Helios 2 support the findings. 235 impacts were recorded in the 5 years time interva1. 83 meteoroid impacts were observed by the ecliptic sensor and 152 by the south sensor. Detailed descriptions of the instrument and the data were published by Grün et al. (1980) and Grün (1981). Most of the particles detected by the ecliptic sensor had masses of $10^{-13}<\mathrm{m}<10^{-10} \mathrm{~g}$. The particles observed by the south sensor had masses $10^{-15^{2}} \lesssim \mathrm{m}^{2} \leqslant 10^{-9} \mathrm{~g}$. This difference has been attributed by Grïn et al. (1980) to two different particle populations, one ("apex" particles) made out of material of normal density $\left(\rho \gtrsim 1 \mathrm{~g} / \mathrm{cm}^{3}\right)$ - only this population of micrometeoroids could be observed by the ecliptic sensor - and another population ("eccentric" particles) of low densities $\rho<1 \mathrm{~g} / \mathrm{cm}^{3}$ which was, in addition, observed by the south sensor. In this paper we concentrate on the 105

R. H. Giese and P. Lamy (eds.), Properties and Interactions of Interplanetary Dust, 105-111.

(C) 1985 by D. Reidel Publishing Company. 
spatial and orbital distributions of micrometeoroids observed by Helios.

\section{FLUX DISTRIBUTION}

The radial distribution of the dust flux could only be derived after a long integration time. Fig. 1 shows the result for both sensors. Since
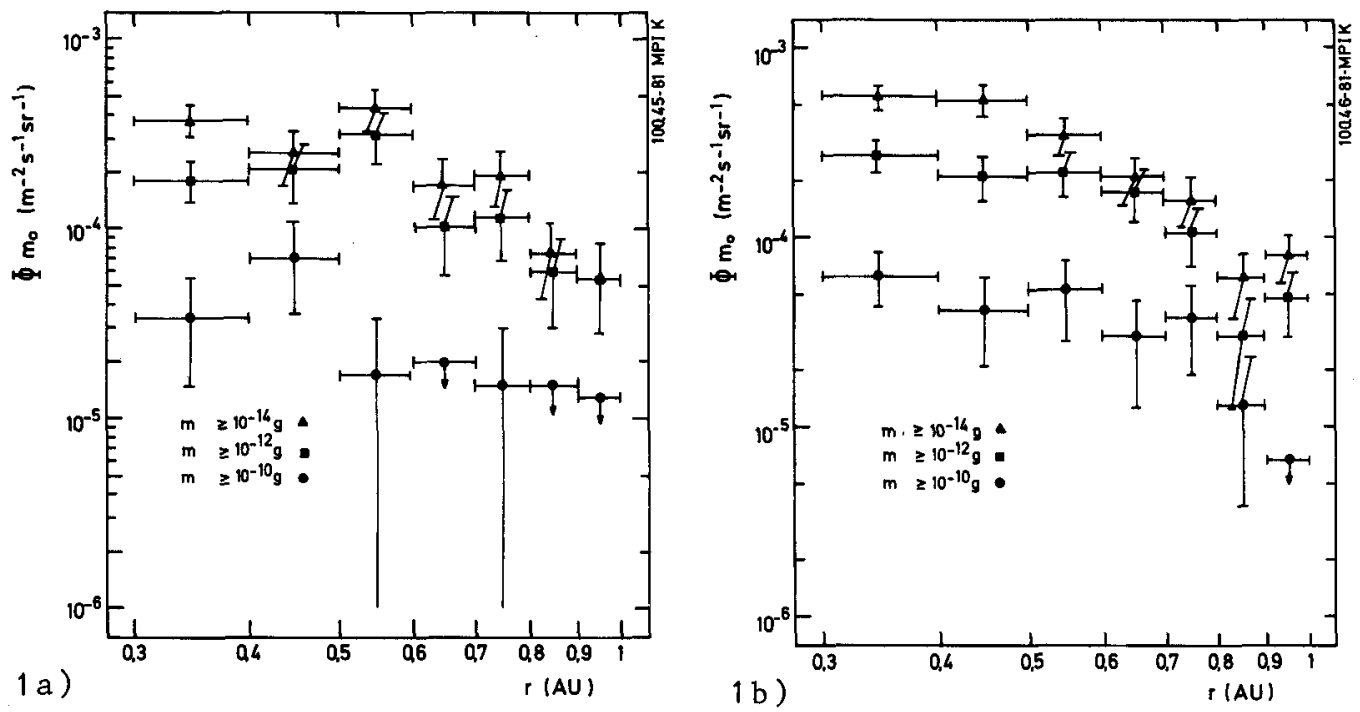

Fig. 1: Radial variation of the micrometeoroid flux observed by Helios. The fluxes were averaged over a $0.1 \mathrm{AU}$ interval. The statistical errors correspond to the particle numbers observed. Upper limits are shown where no particles were detected. The fluxes given for $\mathrm{m} \geq 10^{-12} \mathrm{~g}$ and $10^{-14} \mathrm{~g}$ are lower limits since the instrument did not record particles impacting at speeds $v \leqslant 5 \mathrm{~km} / \mathrm{s}$ and $20 \mathrm{~km} / \mathrm{s}$, respectively.
a) ecliptic sensor
b) south sensor

Helios is a spinning spacecraft the fluxes shown are averaged over all directions in the ecliptic plane. Flux values have been calculated from the number of impacts detected during the corresponding time intervals and by taking into account the sensitive areas of $54.5 \mathrm{~cm}^{2}$ and $66.5 \mathrm{~cm}^{2}$ and effective solid angles of $1.04 \mathrm{sr}$ and $1.39 \mathrm{sr}$ for the ecliptic and south sensor, respectively. Both sensors measured fluxes increased by a factor between 3 and 10 going inward from 1 to $0.3 \mathrm{AU}$. However, there is a significant difference in the radial variation of the fluxes measured by both sensors. Besides a somewhat reduced $f 1 u x$ in the radial interval 0.8 to $0.9 \mathrm{AU}$ ( $\mathrm{m} \geq 10^{-12}$ and $10^{-14} \mathrm{~g}$ ) compared to both adjacent intervals the flux onto the south sensor is steadily increasing towards the sun. This is compatible with the steady increase in zodiacal light intensity reported by Leinert et al. (1981). In contrast to that, the flux recorded 
by the ecliptic sensor has its maximum $\left(\mathrm{m}>10^{-12}\right.$ and $\left.10^{-14} \mathrm{~g}\right)$ in the radius interval between 0.5 and $0.6 \mathrm{AU}$ and stays constant or even decreases further in.

\section{AZIMUTHAL DISTRIBUTTON}

During one spin revolution of the Helios spacecraft each sensor scans a full circle in azimuth. Fig. 2 shows the azimuthal distribution of impacts on both sensors in various distance intervals. Outside $0.6 \mathrm{AU}$

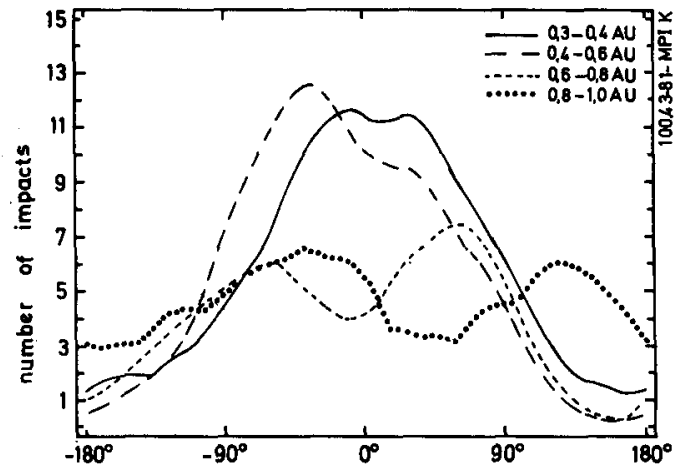

2a)

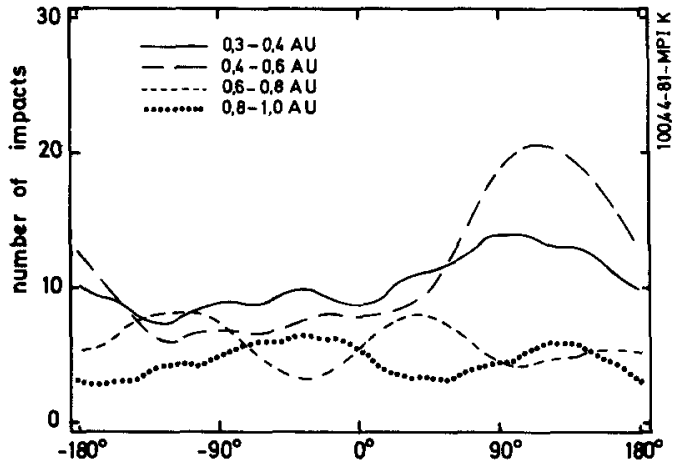

2b)

Fig. 2: Azimuthal distribution of impacts. An individual impact is represented by the angular probability distribution centered on the actual sensor pointing direction. Apex direction $\approx 0^{\circ}$, solardirection $=90^{\circ}$. Azimuthal distributions are shown for 4 different radius intervals.
a) ecliptic sensor
b) south sensor

the azimuthal distributions are generally flat with perhaps a slight enhancement around the apex direction $\left(0^{\circ}\right)$. Inside 0.6 AU the azimutha1 distribution from ecliptic sensor is strongly peaked towards the apex direction. Similar distributions have been observed by the micrometeoroid experiments on board the Pioneer 8 and 9 spacecrafts (Berg and Gerloff, 1971) for big particles and the HEOS-2 satellite (Hoffmann et al., 1975). A possible source of these "apex" particles has been discussed by Grün and Zook (1980). A quite different azimuthal distribution has been detected by the south sensor inside $0.6 \mathrm{AU}$. The number of impacts from the apex direction is smaller than that observed by the ecliptic sensor. This indicates that the average inclination of "apex" particles is smaller than $30^{\circ}$. Most of the impacts were detected when the south sensor was pointing approximately in the solar direction $\left(90^{\circ}\right)$. An even stronger increase of the dust flux from the solar direction has been observed by the Pioneer 8 and 9 experiments for small particles (Berg and Grün, 1973). Zook and Berg (1975) interpreted the "solar" particles to be small fragment particles ( $\beta$-meteoroids) leaving the solar system 
on hyperbolic orbits. The number of $\beta$-meteoroids observed by Helios is much smaller than that detected by the Pioneer spacecrafts.

\section{IMPACT SPEED DISTRIBUTION}

The speed of micrometeoroids impacting the Helios detectors has been determined. There is a bias for small particles $\left(\mathrm{m} \lesssim 10^{-12} \mathrm{~g}\right.$ ) towards high speeds and for big particles $\left(\mathrm{m}>10^{-12} \mathrm{~g}\right.$ ) towards ${ }^{2}$ 1ow speeds. Small particles with low speeds are not detectable and big particles with high speeds cause saturation of the measurement and no speed value can be derived. In the mass range $10^{-13} \mathrm{~g}<\mathrm{m}<10^{-10} \mathrm{~g}$ no bias exists for intermediate impact speeds $10 \mathrm{~km} / \mathrm{s}<\overline{\mathrm{v}} \leq \overline{20} \mathrm{~km} / \mathrm{s}$. Fig. $3 \mathrm{a}$ shows the radial speed distribution for particles observed by the ecliptic sensor. The average impact speed rises from $7 \mathrm{~km} / \mathrm{s}$ in the interval 0.9 to $1 \mathrm{AU}$ to $23 \mathrm{~km} / \mathrm{s}$ at 0.3 to $0.4 \mathrm{AU}$.

With the south sensor the highest average impact speed ( $19 \mathrm{~km} / \mathrm{s})$ in the mass range $10^{-13}<\mathrm{m}<10^{-10} \mathrm{~g}$ were observed between 0.8 and $1 \mathrm{AU}$, the lowest average speed $(\sim 8 \mathrm{~km} / \mathrm{s})$ at 0.6 to $0.8 \mathrm{AU}$ and further in a steady increase towards $16 \mathrm{~km} / \mathrm{s}$ at 0.3 to 0.4 AU had been measured. In Fig. 3b however we show another subset of the speeds measured with
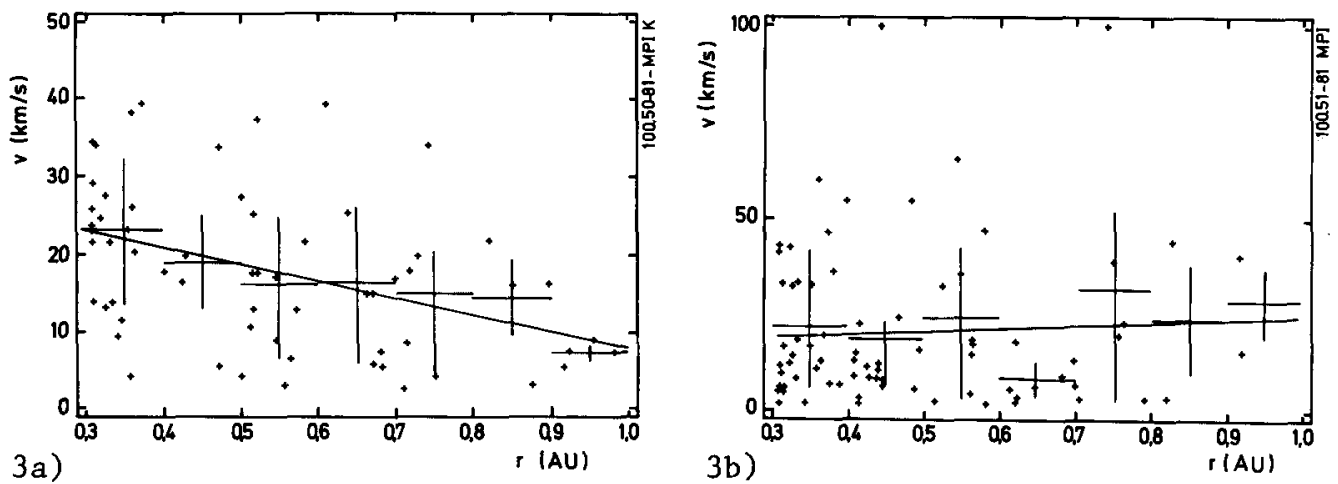

Fig. 3: Radial variation of impact speeds. Small dots are values for individual particles. Crosses are average values over $0.1 \mathrm{AU}$ intervals with $68 \%$ of the measurements lying within the error bars. Also a linear fit through all measurements is shown.

a) particle with masses $10^{-13} \leq \mathrm{m} \leq 10^{-10} \mathrm{~g}$ recorded by the ecliptic sensor;

b) "eccentric" particles observed by the south sensor.

the south sensor. Particles on "eccentric" orbits have been identified by Grün et al. (1980) to be those which are only recorded by the south sensor and not by the ecliptic sensor. These particles have the highest average impact speeds $(\sim 30 \mathrm{~km} / \mathrm{s})$ at $1 \mathrm{AU}$ and slightly lower $(\sim 20 \mathrm{~km} / \mathrm{s})$ 
at $0.3 \mathrm{AU}$. Such a speed variation together with the flux variation observed by the south sensor is compatible with the spatial density variation determined by Leinert et a1. (1981) according to $n \sim r^{-1.3}$. The "apex" particles observed by the ecliptic sensor show a quite different radial spatial density profile. Their spatial density has its maximum at 0.5 to $0.6 \mathrm{AU}$ and decreases both inwards and outwards.

\section{ORBITAL DISTRIBUTION}

From the measured impact speed, the actual pointing direction of the sensor and the position and velocity of the Helios space probe, the heliocentric velocity of micrometeoroids can be determined. Because of the large field-of-view of the sensors and the large uncertainty of the impact speed measurement only probability distributions for the direction and the amount of the velocity vector are obtained. These distributions are transformed into probability distributions of orbital elements for each individual particle, taking into account its radiation pressure constant $\beta$. For a more detailed description of the method and its biases see Schmidt and Grün $(1979,1980)$. Fig. 4 shows the pro-
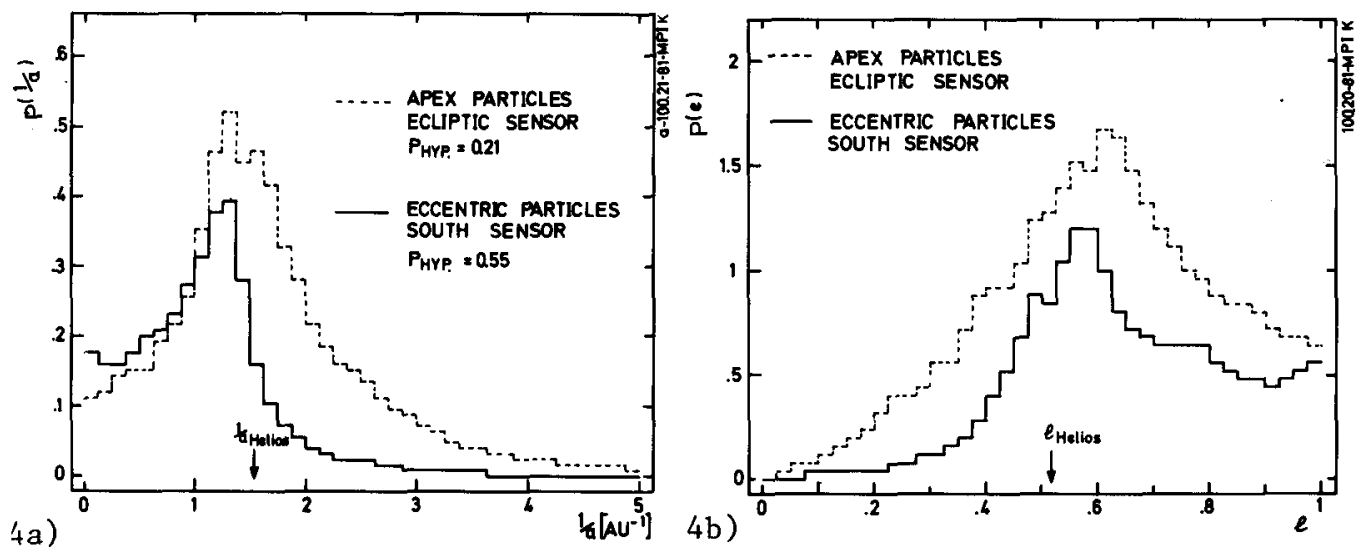

Fig. 4: Probability distribution of orbital elements for "apex" particles and "eccentric" particles. The total probabilities are normalized to 1 . The strong enhancement of the probability at the values of the orbital elements of Helios is a bias involved with this method.

a) distribution of the reciprocal semi-major axes. The probability for hyperbolic orbits $\mathrm{p}_{\mathrm{HYP}}$ is indicated;

b) distribution of eccentricities.

bability distributions of orbital elements for the two different populations of micrometeoroids observed by Helios: "apex"-particles and 
"eccentric" particles. Because of the biases involved with this method only relative results will be discussed here.

The "apex" particles show a much higher probability for bound orbits than for hyperbolic orbits ( particles have maximum probability for hyperbolic orbits $\left(\mathrm{p}_{\mathrm{HYP}}=0.55\right)$. However, the absolute value of this probability will be too high due to a methodical bias. The average reciprocal semi-major axes (Fig. 4a) of "apex" particles is larger than that of Helios $\left(1 / \mathrm{a}\right.$ Helios $=1.54 \mathrm{AU}^{-1}$ ) in contrast to that of "eccentric" particles which is smaller. "Apex" particles which constitute about $60 \%$ of all observed micrometeoroids are on low energy orbits ( $\bar{a} \leqslant 0.6 \mathrm{AU})$, whereas $30 \%$ of them ("eccentric" particles) are on high energy orbits $(\bar{a} \geq 0.9 \mathrm{AU})$. At least $10 \%$ of the observed particles show high probabilities for travelling on hyperbolic orbits.

The probability distributions of the eccentricities are displayed in Fig. 4b. Both distributions have maxima and means above the value of Helios (e $e_{\text {Helios }}=0.52$ ). This shows that likewise the "apex" particles move on high eccentric orbits with an average eccentricity of $\bar{e}=0.6$

\section{REFERENCES}

Berg O.E. and Gerloff U. (1971) "More than two years of micrometeorite data from two Pioneer satellites", Space Res. XI, 225.

Berg O.E. and Grün E. (1973) "Evidence of hyperbolic cosmic dust particles", Space Res. XIII, 1047.

Grün E. (1981) "Physikalische und chemische Eigenschaften des interplanetaren Staubes - Messungen des Mikrometeoritenexperiments auf Helios", Bundesministerium für Forschung und Technologie, Forschungsbericht W 81-034.

Grün E., Pailer N., Fechtig H. and Kissel J. (1980) "Orbital and physical characteristics of micrometeoroids in the inner solar system as observed by Helios 1", Planet. Space Sci., 28, 333.

Grün E. and Zook H.A. (1980) "Dynamics of micrometeoroids in the inner solar system", in: 'Solid Particles in the Solar System' (Eds. I. Halliday and B.A. McIntosh), Reide1, Dordrecht, 293.

Hoffmann H.J., Fechtig H., Grün E. and Kisse1 J. (1975) "Temporal fluctuations and anisotropy of the micrometeoroid flux in the EarthMoon system", Planet. Space Sci. 23 3 , 985 .

Leinert C., Richter I., Pitz E. and Planck B. (1981) "The zodiacal light from 1.0 to $0.3 \mathrm{AU} "$, Astron.Astrophys. $10 \underline{\underline{0}} \underline{=}, 177$.

Schmidt K.D. and Grün E. (1979) "The distribution of orbita1 elements of interplanetary dust in the inner solar system as detected by the Helios spaceprobe", Space Res. XIXI 439. 
Schmidt K.D. and Grün E. (1980) "Orbital elements of micrometeoroids detected by the Helios 1 spaceprobe in the inner solar system", in: 'Solid Particles in the Solar System' (Eds. I. Halliday and B.A. Mc(ntosh), Reide1, Dordrecht, 321.

Zook H.A. and Berg O.E. (1975) "A source for hyperbolic cosmic dust particles", Planet. Space Sci. 23 , 183. 